A New Class of Non-Linear,

Finite-Volume Methods for

Vlasov Simulation

J. W. Banks, J. A. Hittinger

December 1, 2009

IEEE Transactions on Plasma Science 
This document was prepared as an account of work sponsored by an agency of the United States government. Neither the United States government nor Lawrence Livermore National Security, LLC, nor any of their employees makes any warranty, expressed or implied, or assumes any legal liability or responsibility for the accuracy, completeness, or usefulness of any information, apparatus, product, or process disclosed, or represents that its use would not infringe privately owned rights. Reference herein to any specific commercial product, process, or service by trade name, trademark, manufacturer, or otherwise does not necessarily constitute or imply its endorsement, recommendation, or favoring by the United States government or Lawrence Livermore National Security, LLC. The views and opinions of authors expressed herein do not necessarily state or reflect those of the United States government or Lawrence Livermore National Security, LLC, and shall not be used for advertising or product endorsement purposes. 


\title{
A New Class of Non-Linear, Finite-Volume Methods for Vlasov Simulation
}

\author{
Jeffrey W. Banks and Jeffrey A. F. Hittinger
}

\begin{abstract}
Methods for the numerical discretization of the Vlasov equation should efficiently use the phase space discretization and should introduce only enough numerical dissipation to promote stability and control oscillations. A new high-order, non-linear, finite-volume algorithm for the Vlasov equation that discretely conserves particle number and controls oscillations is presented. The method is fourth-order in space and time in wellresolved regions, but smoothly reduces to a third-order upwind scheme as features become poorly resolved. The new scheme is applied to several standard problems for the Vlasov-Poisson system, and the results are compared with those from other finitevolume approaches, including an artificial viscosity scheme and the Piecewise Parabolic Method. It is shown that the new scheme is able to control oscillations while preserving a higher degree of fidelity of the solution than the other approaches.
\end{abstract}

Index Terms-Vlasov equation, plasma simulation, finitevolume methods.

\section{INTRODUCTION}

The Vlasov equation is a fundamental kinetic model for lowdensity, high-temperature plasmas typical of many plasmas of interest. Because this model expresses the particle distribution as a function of time, particle location, and particle velocity, direct discretization methods are extremely expensive; the computational cost increases geometrically with dimension. Thus, stochastic Particle-In-Cell (PIC) methods [1] have been the dominant Vlasov simulation technique. Continuum (or Eulerian) discretizations of Vlasov are still useful in a complementary role to PIC, since the continuum approach can provide information where the inherent noise of PIC may mask physical effects.

Development of continuum discretization techniques for the Vlasov equation has not received the attention it deserves, perhaps because available computer resources have been insufficient to simulate meaningful multidimensional problems. Much work that has been done has focused on the dimensionally-split, semi-Lagrangian approach, with a variety of spline or spectral interpolants [2], [3], [4] used. Shortcomings of this approach include the lack of discrete conservation, the occurrence of unphysical oscillations in the solution, and the generation of negative values in the positivedefinite solution. Several variations of the semi-Lagrangian approach have been developed to address these numerical difficulties [5], [6], [7], [8], [9]. Nevertheless, in the modern era of massively parallel computing, one of the primary advantages of the semi-Lagrangian approach - the lack of a

J.W. Banks and J.A.F. Hittinger, Center for Applied Scientific Computing, Lawrence Livermore National Laboratory, Livermore, California 94551, banks20@1lnl.gov, hittinger1@1lnl.gov stability restriction on the time step used - is diminished by the fact that domain decomposition favors local schemes of compact support. Furthermore, split algorithms are inherently task serial, whereas unsplit algorithms are more amenable to task parallelization on multicore processors.

In the last decade, as increased computer power has enabled Vlasov simulation in higher-dimensions, attention has been drawn towards discretization methods developed in the applied mathematics and engineering communities for hyperbolic systems. Examples include finite-element [10] and pseudospectral methods [11] that allow for adaptive mesh refinement, as well as finite-volume methods from compressible gas dynamics [12], [13], [14]. In related work, 5D gyrokinetic core codes have been developed using low- and high-order, linear finite-difference schemes [15], [16], [17].

A very promising class of such algorithms are the fluxbased, higher-order, non-linear finite-volume schemes [18], [19], [20] that can enforce conservation, monotonicity, and, with further modification, positivity; at least one 4D VlasovMaxwell code based on the Piecewise Parabolic Method (PPM) has been developed [14]. Additional advantages of these finite-volume approaches are that they can easily be extended to higher-order in both space and time and that they naturally fit within the framework of adaptive mesh refinement (AMR). Both higher-order and AMR can be used to reduce the cost of continuum Vlasov simulation. However, nonlinear finite-volume methods have disadvantages for Vlasov simulation as well, most notably, a potentially severe stability restriction on time step size and increased computational cost due to the oscillation control.

In this paper, we present a new class of non-linear finitevolume schemes that attempt to balance these trade-offs. The new approach is based on the higher-order finite-volume framework developed in [21], [22] and has certain similarities with the more standard WENO approach. However, this new scheme is optimized more for Vlasov solutions than for the shock-capturing applications for which PPM and WENO were originally developed. The result is a scheme that does an excellent job of preserving order while adding sufficient dissipation to control unphysical oscillations. An optional addition to the algorithm based on multidimensional flux-corrected transport (FCT) algorithm [23] can be used to self-consistently enforce distribution function positivity [21].

In the next section, we briefly describe the Vlasov-Poisson system we use as our model problem. We then present the generic formulation of the fourth-order, finite-volume discretization. In Section III, we discuss the challenges of Vlasov simulation within the context of the numerical analysis 
of methods for hyperbolic partial differential equations and follow with a discussion of traditional fixes to these problems. We then describe the new algorithm in full detail, and in Section VII we present the results of comparative numerical studies using the new algorithm.

\section{Governing Equations and Model Problem}

Because the purpose of the present work is to describe a new algorithm and demonstrate its performance, it is appropriate to describe a simple physical model. Consider a collisionless quasi-neutral plasma in one space and one velocity dimension where the ions have been assumed to be stationary. As a further simplification, assume that we are in the non-relativistic, zeromagnetic field limit. For this case, the well-known VlasovPoisson system (1)-(3) describes the evolution of the electron distribution function $f(x, v, t)$ in phase space $(x, v)$ :

$$
\begin{gathered}
\frac{\partial}{\partial t} f(x, v, t)+v \frac{\partial}{\partial x} f(x, v, t)-E \frac{\partial}{\partial v} f(x, v, t)=0, \\
E(x, t)=-\frac{\partial}{\partial x} \phi(x, t), \\
\frac{\partial^{2} \phi}{\partial x^{2}}=\int_{-\infty}^{\infty} f(x, v, t) d v-1 .
\end{gathered}
$$

In equations (1)-(3), $v$ is the velocity, $x$ is the physical coordinate, $t$ is time, $\phi$ is the electric potential, and $E$ is the electric field. We have chosen units such that the electron mass and charge are unity.

We investigate problems on the periodic domain $x \in$ $[-L, L]$ with initial conditions $f(x, v, 0)=f_{0}(x, v)$. The domain is artificially truncated in the $v$ direction at some location $v_{\max }$, and an appropriate outflow/inflow condition is applied. Note that periodicity and (3) imply that

$$
\int_{-L}^{L} \int_{-\infty}^{\infty} f(x, v, t) d v d x=1
$$

for all time. Exact specifications of the initial conditions as perturbations of Maxwellian distributions will be provided as needed.

\section{Motivating ExAMPLE}

In the context of numerical approximation, two prevalent features of Vlasov systems deserve special consideration. The first of these is the fact that the system is non-linear. Although the fourth-order Runge-Kutta integration scheme (RK-4) we adopt here is slightly dissipative, that dissipation is insufficient to stabilize the centered spatial approximations when used for the simulation of sufficiently non-linear problems [24]. The question of whether the Vlasov systems of interest are in this class is not proved, but practical experience indicates quite strongly that additional dissipation of some kind is required, while respecting certain conservation properties. The second feature to which we need to pay heed is the shearing nature of the solutions. By this we mean that because the spatial advection velocity is the velocity coordinate, structures present in the initial conditions will tend to shear and become thin as time progresses.

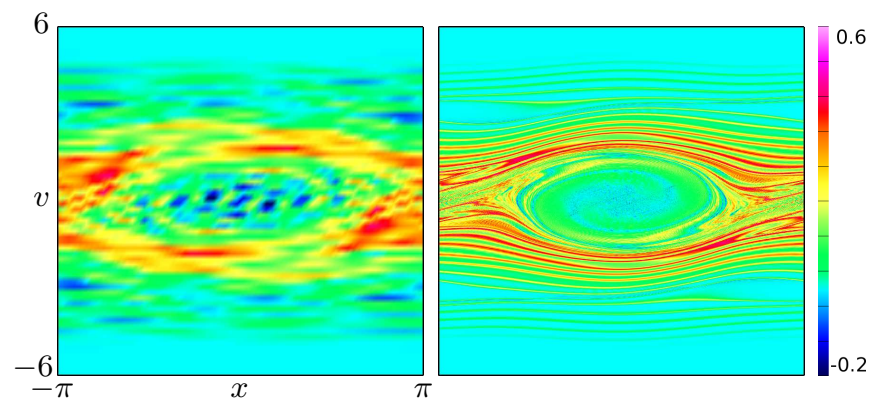

Fig. 1. Examples showing the need for artificial viscosity. Plotted is the distribution function $f(x, v, t)$ at time $t=45$ computed using $N_{x}=N_{v}=$ 64 (left) and $N_{x}=N_{v}=2048$ (right).

These two features are demonstrated using a two-stream instability problem using the parameters from [4] but a stronger initial spatial perturbation. The initial distribution function used here is given by

$$
f(x, v, t=0)=\frac{v^{2}}{\sqrt{2 \pi}}\left(1-\frac{1}{2} \cos \left(\frac{x}{2}\right)\right) \exp \left(-\frac{v^{2}}{2}\right),
$$

and the domain is given by $L=2 \pi$ and $v_{\max }=6$. Figure 1 presents numerically computed results using the linear, centered scheme of Section V. Shown are a coarse simulation $\left(N_{x}=N_{v}=64\right)$ and a finely resolved simulation $\left(N_{x}=N_{v}=2048\right)$ at the same time $t=45$. Both results capture, at some level, the broad dynamics of the problem, but for both cases the approximations exhibit erroneous numerical oscillations. These are caused by some combination of fine scales in the exact solution and the inherent non-linearity of the governing system. Notice further that for both simulations the electron number density drops significantly below zero. These results serve to demonstrate that poor behavior can be exhibited by schemes using purely central spatial discretizations for this type of non-linear problem.

\section{Possible Fixes}

Numerical analysis informs us that most low-dissipation linear discretizations applied to variable-coefficient and nonlinear hyperbolic problems will eventually generate spurious oscillations and often become unstable [24]. The problem arises from the nature of the continuous solutions that typically generate finer and finer scales, and an accurate discrete approximation will do the same. Thus, the discrete operator eventually generates scales unresolvable on the mesh and oscillations are produced. From a spectral perspective, the energy in unresolvable modes is aliased to resolvable modes.

Since the earliest days of numerical simulation, researchers have wrestled with this problem. The generation of oscillations is a signal that the solution is under-resolved, and one philosophy is that the computation should be terminated or a finer mesh used. This is often impractical, and if the under-resolved scales have little influence on the goal of the calculation, increasing the resolution would be wasteful. Many other approaches have emerged, and the commonality is that these methods attempt to remove energy either directly or indirectly from under-resolved scales through some sort of dissipation. The trade-off is that the accuracy of some scales 


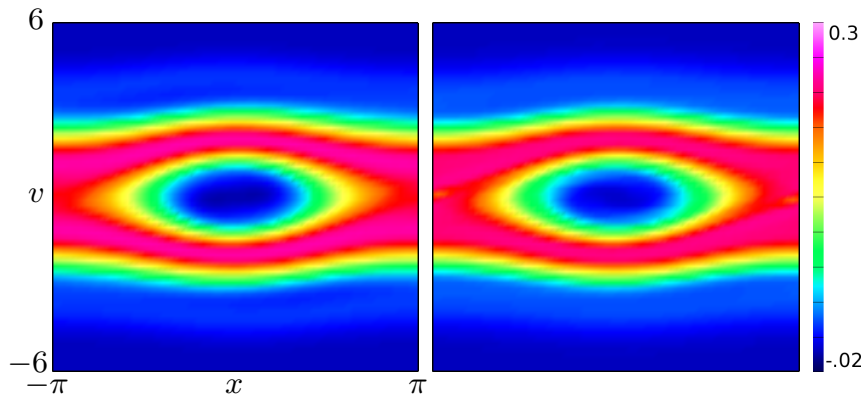

Fig. 2. Distribution function $f(x, v, t)$ at time $t=45$ for the two-stream instability problem using the mesh $N_{x}=N_{v}=64$ and the centered scheme with artificial viscosity (left) and PPM (right).

resolvable on the grid is sacrificed. Thus, while each of these "fixes" can be posed such that particle number is conserved to round-off error, all other conserved quantities are accurate only to within truncation error.

The simplest solution is to add a linear dissipative term to the discretization in the form of an artificial viscosity or hyperviscosity [24]. Precise forms are known that guarantee stability [24]. However, there are drawbacks to this approach. First, the linear viscosity term is always active and so continuously damps all modes in the solution, which can even smear well-represented profiles. In addition, high-derivative hyperviscosity terms are required to achieve higher-order, but discretizations of higher derivatives are often not robust. Finally, the method introduces an adjustable coefficient on which the discrete solution depends.

A related approach from spectral discretizations are dealiasing [25, §11.5] or direct filtering [5]. Here, the coefficients of a predetermined set of the high-wavenumber modes is zeroed out at the end of each time step. Effectively, energy that naturally flows into these modes is artificially removed from the system.

Shock-capturing methods are a somewhat different class of schemes that have been developed in computational compressible fluid dynamics. A well-known theorem [26] states that linear, monotone algorithms for hyperbolic equations will be at most first-order. To achieve higher-order, shockcapturing approaches non-linearly adapt the stencil and order of the discretization in order to obtain monotone or nearly monotone solutions. Standard methods include flux-limiting methods of the Flux-Corrected Transport (FCT) type [23], [27] and geometric approaches based on limiting conservative interpolations within cells, such as the piecewise linear MUSCL scheme [28] and the PPM scheme [18]. Results using a method-of-lines variant of the PPM scheme [22] are shown in Figure 2. In a method-of-lines approach this scheme is fourth-order accurate in space and, for our RK-4 time discretization, is fourth-order accurate in time. A difficulty with these methods is that, because they are optimized for shock-capturing, they all reduce to first-order in regions where the solution is under-resolved and typically also at solution extrema, although recent work has tried to minimize extrema clipping [19].

Other geometrically-inspired schemes include the (weighted) essentially non-oscillatory ((W)ENO) [20] methods. These methods do not guarantee monotonicity, but they are higher-order, do not clip extrema, and do a reasonable job of minimizing oscillations, even around a discontinuity. Schemes of this type adapt their stencil in order to obtain the smoothest interpolant of the data. In the standard upwind formulation, WENO schemes select a computational stencil as a weighted combination from a collection of upwind-biased, lower-order stencils, e.g. a fifth-order discretization in smooth regions composed of three third-order stencils. On the other hand, switching from from central to upwind stencils may have advantages if the goal is to switch from a dissipationless difference to a difference with implicit numerical dissipation. It is this fact that leads to our new approach in Section VI.

Before proceeding, however, we will make one final point about positivity. Unphysical oscillations are the most obvious cause of non-positive solution values. However, merely controlling oscillations does not guarantee solution positivity, particularly in multiple dimensions. Again, theory demonstrates that there are no linear schemes above first-order that preserve solution positivity [26], and the non-linear oscillationcontrolling schemes above do not by themselves guarantee positivity. To retain positivity, one must appeal to some other mechanism. By far, the most common approach is to floor non-positive values to zero; this technique is neither consistent with the governing equations nor is conservative.

Alternatively, as discussed in [21], a consistent, conservative correction to enforce solution positivity can be formulated using FCT. Specifically, Zalesak's multidimensional FCT scheme [23] allows for the imposition of constraints on the solution other than monotonicity. In this usage, the multidimensional FCT scheme limits base-scheme fluxes with positivitypreserving fluxes just enough to guarantee the updated solution is positive definite. Any base-scheme fluxes can be used, including fluxes previously limited by other means; the procedure works with all of the linear and non-linear discrete fluxes discussed in this paper. Thus, this FCT approach decouples the issues of oscillation control and positivity preservation, while the flux-based form ensures consistency and conservation.

We mention the FCT positivity-preservation procedure here for completeness. We have made successful use of the approach in practice with all of the methods presented in this paper. However, the intent of this manuscript is to focus on the properties of our new oscillation-suppressing scheme in contrast to other common methods. In the interest of space, and since the FCT positivity-preservation procedure is independent of the choice of base-scheme flux, we have elected to include no results using the FCT algorithm in this paper. The interested reader is referred to [21] for some comparative results of the FCT scheme for positivity preservation.

\section{BASIC NUMERICAL METHOD}

Our basic finite-volume discretizations follows the approach in [21], [22]. Let us rewrite the Vlasov equation (1) in fluxdivergence form:

$$
\frac{\partial}{\partial t} f(\xi, t)+\nabla_{\xi} \cdot F(f, \xi, t)=0,
$$


where the phase-space flux vector is $F=\left(F_{x}, F_{v}\right)=a f$, the phase-space velocity vector is $a=(v,-E)$, and the divergence is with respect to $\xi=(x, v)$. We construct a uniform Cartesian partitioning of phase space into control volumes,

$$
V_{i j}=\left[i-\frac{1}{2}, i+\frac{1}{2}\right] \Delta x \times\left[j-\frac{1}{2}, j+\frac{1}{2}\right] \Delta v .
$$

Integrating (5) over one such control volume and dividing by the volume $\Delta x \Delta v$, we obtain the exact system of ordinary differential equations

$$
\begin{aligned}
\frac{d}{d t} \bar{f}_{i j}= & -\frac{1}{\Delta x \Delta v} \int_{V_{i j}} \nabla_{\xi} \cdot F d x d v \\
= & -\frac{1}{\Delta x}\left(\left\langle F_{x}\right\rangle_{i+\frac{1}{2}, j}-\left\langle F_{x}\right\rangle_{i-\frac{1}{2}, j}\right) \\
& -\frac{1}{\Delta v}\left(\left\langle F_{v}\right\rangle_{i, j+\frac{1}{2}}-\left\langle F_{v}\right\rangle_{i, j-\frac{1}{2}}\right),
\end{aligned}
$$

where the cell average $\bar{f}_{i j}$ is defined to be

$$
\bar{f}_{i j} \equiv \frac{1}{\Delta x \Delta v} \int_{V_{i j}} f d x d v,
$$

and the angle braces denote face averages, for example,

$$
\left\langle F_{x}\right\rangle_{i+\frac{1}{2}, j}=\frac{1}{\Delta v} \int_{v_{j-1 / 2}}^{v_{j+1 / 2}} F\left(x_{i+1 / 2}, v\right) d v .
$$

The face-averaged fluxes can be approximated by the products of other face-averaged quantities and transverse derivatives by using Taylor series expansions. Define the second-order central difference operators $D_{x} u_{i j} \equiv u_{i+1, j}-u_{i-1, j}$ and $D_{v} u_{i j} \equiv$ $u_{i, j+1}-u_{i, j-1}$. Then, to fourth-order,

$$
\begin{aligned}
\left\langle F_{x}\right\rangle_{i+\frac{1}{2}, j} & \approx\langle v\rangle_{i+\frac{1}{2}, j}\langle f\rangle_{i+\frac{1}{2}, j}+\frac{1}{48} D_{v}\langle f\rangle_{i+\frac{1}{2}, j}, \\
\left\langle F_{v}\right\rangle_{i, j+\frac{1}{2}} & \approx-\langle E\rangle_{i, j+\frac{1}{2}}\langle f\rangle_{i, j+\frac{1}{2}} \\
& -\frac{1}{48} D_{x}\langle E\rangle_{i, j+\frac{1}{2}} D_{x}\langle f\rangle_{i, j+\frac{1}{2}} .
\end{aligned}
$$

Relating the face averages of $a$ and $f$ to cell averages of the same quantities completes the spatial discretization. For comparison in subsequent sections, the baseline linear, central, fourth-order approximation is used, e.g.,

$$
\langle f\rangle_{i+\frac{1}{2}, j} \approx \frac{7}{12}\left(\bar{f}_{i, j}+\bar{f}_{i+1, j}\right)-\frac{1}{12}\left(\bar{f}_{i-1, j}+\bar{f}_{i+2, j}\right) .
$$

One variant we use for comparison is the addition of a linear, artificial viscosity (AV) that adds $O\left(\Delta x^{4}\right)$ and $O\left(\Delta v^{4}\right)$ dissipation to the truncation error, that is,

$$
\begin{aligned}
\langle f\rangle_{i+\frac{1}{2}, j}^{A V} & =\langle f\rangle_{i+\frac{1}{2}, j} \\
& -\mu \Delta x\left[\bar{f}_{i+2, j}-3\left(\bar{f}_{i+1, j}-\bar{f}_{i, j}\right)-\bar{f}_{i-1, j}\right],
\end{aligned}
$$

with a constant $\mu>0$; the form is similar for $v$-faces. A choice of $\mu=0.1$ performs reasonably well for the problems considered in this paper. When differenced in fluxdivergence form (6), the additional terms (8) approximate fourth-derivatives of the solution in each coordinate direction. We will also compare with the non-linear, method-of-lines PPM scheme described in detail in [22]. The choice of the face average approximation is what distinguishes our new scheme from previous work.
To compute the phase-space velocity, we require velocityface-averages of the electric field; these are equivalent to the configuration-space cell-averages of the electric field computed by solving the potential equation. The instantaneous cell-averaged electric field are to fourth-order:

$$
\bar{E}_{i} \approx \frac{1}{12 \Delta x}\left[8\left(\bar{\phi}_{i+1}-\bar{\phi}_{i-1}\right)-\bar{\phi}_{i+2}-\bar{\phi}_{i-2}\right] .
$$

In configuration space, we average (3) over each configurationspace cell $V_{i}$ :

$$
\frac{1}{\Delta x} \int_{V_{i}} \frac{\partial^{2} \phi(x, t)}{d x^{2}} d x=\bar{\rho}_{i}(t) .
$$

Discretely, we construct a nearly pentadiagonal system from the stencil

$$
30 \bar{\phi}_{i}-16\left(\bar{\phi}_{i+1}+\bar{\phi}_{i-1}\right)+\left(\bar{\phi}_{i+2}+\bar{\phi}_{i-2}\right)=12 \Delta x \bar{\rho}_{i},
$$

which gives a fourth-order approximation of the cell averaged potential. The resulting linear algebra problem can be LUdecomposed once at the beginning of a run and stored. Periodic boundary conditions in $x$ lead to a singular system, which is a well-known problem that can be handled by projecting out the portion of $\bar{\rho}(x)$ residing in the null space of the matrix. This amounts to ensuring that $\sum_{i} \bar{\rho}\left(x_{i}\right)=0$, and in so doing, we ensure that $\bar{\phi}(x)$ is normalized around zero. Of course, since we take a derivative of $\bar{\phi}(x)$ to get $\bar{E}(x)$, the offset has no effect on the solution.

The cell average of net charge density is computed in this finite-volume formulation:

$$
\begin{aligned}
\rho(x, t) & =1-\int_{-\infty}^{\infty} f(x, v, t) d v \\
& =1-\Delta v \sum_{j=-\infty}^{\infty} \bar{f}_{i j} \approx 1-\Delta v \sum_{j=-v_{\max }}^{v_{\max }} \bar{f}_{i j} .
\end{aligned}
$$

The last approximation occurs in any non-infinite discretization basis; we adopt the standard approach of truncating the velocity domain to $|v| \leq v_{\max }$, where the number of particles beyond this domain is treated as negligible.

For the temporal discretization of the semi-discrete Vlasov equation (6), any stable method can be used. We do not have competing time scales in this problem, so as in [22], we choose the standard explicit fourth-order Runge-Kutta scheme. At each stage in the Runge-Kutta update, we solve the discrete potential equation (9) prior to evaluating the phase-space flux divergence as given by the right-hand side of (6).

\section{Vi. A New Numerical Method}

We wish to devise a numerical method that has the property that, for well-represented solutions, the fourth-order, centered approximation (7) is used, but that introduces numerical dissipation when solution features cannot be represented on a given mesh. We take the stance that a suitable viscosity is provided by the third-order, upwind approximation, and our goal is to derive a solution-dependent switch to transition smoothly between the fourth order central and third order upwind fluxes. As an additional design criteria, we seek to preserve the discretization stencil of the centered, fourth-order approximation. 
We focus on the determination of the face average $F_{i+\frac{1}{2}, j}$; the other face averages are determined in a similar manner. We suppress mention of the time step for clarity. Similarly to the WENO method [20], we compose the face reconstruction as a weighted sum of third order approximations:

$$
\langle F\rangle_{i+\frac{1}{2}, j} \approx w_{i+\frac{1}{2}, j, L}\langle F\rangle_{i+\frac{1}{2}, j, L}+w_{i+\frac{1}{2}, j, R}\langle F\rangle_{i+\frac{1}{2}, j, R},
$$

with

$$
\langle F\rangle_{i+\frac{1}{2}, j, L} \approx \frac{1}{6}\left(-\bar{f}_{i-1, j}+5 \bar{f}_{i, j}+2 \bar{f}_{i+1, j}\right)
$$

and

$$
\langle F\rangle_{i+\frac{1}{2}, j, R} \approx \frac{1}{6}\left(2 \bar{f}_{i, j}+5 \bar{f}_{i+1, j}-\bar{f}_{i+2, j}\right) .
$$

Here $\langle F\rangle_{i+\frac{1}{2}, j, L}$ and $\langle F\rangle_{i+\frac{1}{2}, j, R}$ are third-order approximations of the face average with "L" and "R" indicating data biased to the left or right respectively. Define the ideal weight $d=\frac{1}{2}$ such that for $w_{i+\frac{1}{2}, j, L}=w_{i+\frac{1}{2}, j, R}=d$, equation (10) reduces to the centered fourth order approximation.

Focusing on the stencil associated with $F_{i+\frac{1}{2}, j, L}$, we define the polynomial

$$
P_{i+\frac{1}{2}, j, L}(x)=\frac{A_{i+\frac{1}{2}, j, L}}{2 \Delta x^{2}} \eta^{2}+\frac{B_{i+\frac{1}{2}, j, L}}{2 \Delta x} \eta+C_{i+\frac{1}{2}, j, L},
$$

with

$$
\begin{gathered}
A_{i+\frac{1}{2}, j, L}=\bar{f}_{i+1, j}-2 \bar{f}_{i, j}+\bar{f}_{i-1, j}, \\
B_{i+\frac{1}{2}, j, L}=\bar{f}_{i+1, j}-\bar{f}_{i-1, j}, \\
C_{i+\frac{1}{2}, j, L}=\bar{f}_{i, j} .
\end{gathered}
$$

Here $\eta=x-x_{i}$ measures distance from $x_{i}$. A smoothness indicator in a symmetric interval about $x_{i+\frac{1}{2}}$ is given by

$$
\begin{aligned}
\beta_{i+\frac{1}{2}, j, L} & =\Delta x \int_{0}^{\Delta x}\left(\frac{d}{d \chi} P_{i+\frac{1}{2}, j, L}(\chi)\right)^{2} d \chi \\
& +\Delta x^{3} \int_{0}^{\Delta x}\left(\frac{d^{2}}{d \chi^{2}} P_{i+\frac{1}{2}, j, L}(\chi)\right)^{2} d \chi .
\end{aligned}
$$

This is more concisely written as

$$
\begin{aligned}
& \beta_{i+\frac{1}{2}, j, L}= \\
& \quad \frac{4}{3} A_{i+\frac{1}{2}, j, L}^{2}+\frac{1}{2} A_{i+\frac{1}{2}, j, L} B_{i+\frac{1}{2}, j, L}+\frac{1}{4} B_{i+\frac{1}{2}, j, L}^{2} .
\end{aligned}
$$

Similar reasoning for the right stencil yields

$$
\begin{aligned}
& \beta_{i+\frac{1}{2}, j, R}= \\
& \quad \frac{4}{3} A_{i+\frac{1}{2}, j, R}^{2}-\frac{1}{2} A_{i+\frac{1}{2}, j, R} B_{i+\frac{1}{2}, j, R}+\frac{1}{4} B_{i+\frac{1}{2}, j, R}^{2},
\end{aligned}
$$

where

$$
\begin{gathered}
A_{i+\frac{1}{2}, j, R}=\bar{f}_{i+2, j}-2 \bar{f}_{i+1, j}+\bar{f}_{i, j}, \\
B_{i+\frac{1}{2}, j, R}=\bar{f}_{i+2, j}-\bar{f}_{i, j} .
\end{gathered}
$$

Define approximate stencil weights as

$$
\hat{w}_{i+\frac{1}{2}, j, k}=\frac{a_{i+\frac{1}{2}, j, k}}{a_{i+\frac{1}{2}, j, 1}+a_{i+\frac{1}{2}, j, 2}},
$$

with

$$
a_{i+\frac{1}{2}, j, 1}=\frac{d}{\left(\epsilon+\beta_{i+\frac{1}{2}, j, L}\right)^{2}},
$$

$$
a_{i+\frac{1}{2}, j, 2}=\frac{d}{\left(\epsilon+\beta_{i+\frac{1}{2}, j, R}\right)^{2}},
$$

for $k=1,2$ and $\epsilon$ a small parameter (typically $\epsilon=1 \times 10^{-40}$ ).

As with traditional WENO schemes, convergence rates near certain types of critical points (points with many zero derivatives) may be less than optimal. A detailed discussion of these issues is presented in [29]. As in that work, we perform a mapping of the weights in order to regain optimal convergence rates whenever possible. For our fourth order implementation, the mapping suggested in [29] can be re-written

$$
b_{i+\frac{1}{2}, j, k}=\hat{w}_{i+\frac{1}{2}, j, k}\left(\frac{3}{4}+\hat{w}_{i+\frac{1}{2}, j, k}\left(\hat{w}_{i+\frac{1}{2}, j, k}-\frac{1}{2}\right)\right) .
$$

The final formula for the weights is then

$$
w_{i+\frac{1}{2}, j, k}=\frac{b_{i+\frac{1}{2}, j, k}}{b_{i+\frac{1}{2}, j, 1}+b_{i+\frac{1}{2}, j, 2}} .
$$

Note that we have not associated the weights with either stencil.

The two weights (16) provide a quantitative measure of the degree to which the solution can be represented on the grid. More specifically, the weights define how well the two third-order approximations represent the solution. For smooth flows, they both converge to the ideal weight $d=\frac{1}{2}$ as $O\left(\Delta x^{2}\right)$, and so, in terms of accuracy, it makes no difference which stencil receives which weight. In order to maximize the upwind diffusion in the final numerical method, we choose the larger weight for the upwind, third-order approximation and the smaller weight for the downwind, third-order stencil. Thus,

$$
\text { if } \begin{aligned}
\left(v_{i+\frac{1}{2}, j}>0\right), & \left\{\begin{array}{l}
w_{i+\frac{1}{2}, j, L}=\max _{k}\left(w_{i+\frac{1}{2}, j, k}\right) \\
w_{i+\frac{1}{2}, j, R}=\min _{k}\left(w_{i+\frac{1}{2}, j, k}\right)
\end{array}\right. \\
\text { else } & \begin{cases}w_{i+\frac{1}{2}, j, L} & =\min _{k}\left(w_{i+\frac{1}{2}, j, k}\right) \\
w_{i+\frac{1}{2}, j, R} & =\max _{k}\left(w_{i+\frac{1}{2}, j, k}\right) .\end{cases}
\end{aligned}
$$

The resulting scheme converges at fourth order for smooth flows and uses the same stencil as the linear fourth order algorithm, but introduces an upwind artificial viscosity when the flow features become sharp. Our choice of weights is distinct from the traditional WENO approach, where the weighting is done to favor the smoothest interpolant, even if it is an unstable, downwind approximation.

We return to the two stream example shown in Figures 1 and 2 using the new scheme. The results for $N_{x}=N_{v}=64$ and $N_{x}=N_{v}=2048$ are shown in Figure 3. The effectiveness of the proposed scheme is clear as these results lack the numerical oscillations which characterize the purely centered results of Figure 1. At the same time the scheme captures the relevant features of the solution that are representable on the given computational domain. Notice that for $N_{x}=N_{v}=64$, the solution displays many of the features of the finely resolved computation, even capturing the trapping regions near $( \pm 2, \mp 3)$; this is not seen in either the artificial viscosity or PPM solutions in Figure 2.

To demonstrate the convergence properties of the new method, we consider results for a variable-coefficient advection problem using a manufactured solution. Such an example 

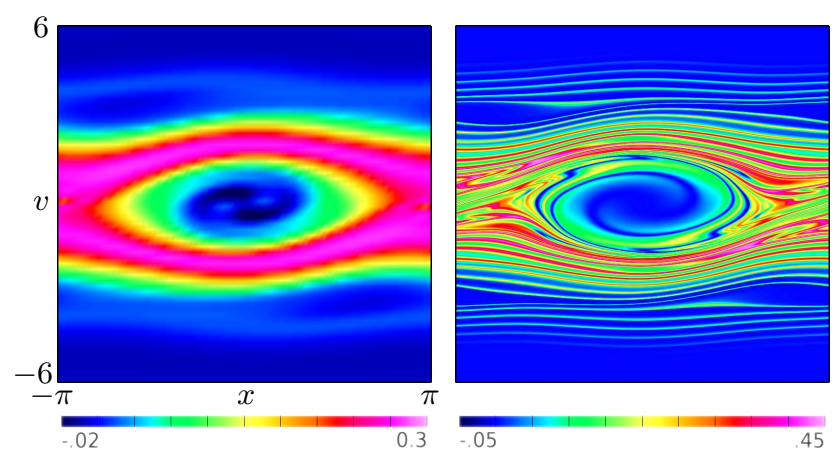

Fig. 3. Distribution function $f(x, v, t)$ at time $t=45$ for the two-stream instability problem. At left are results with $N_{x}=N_{v}=64$ and at right with $N_{x}=N_{v}=2048$.
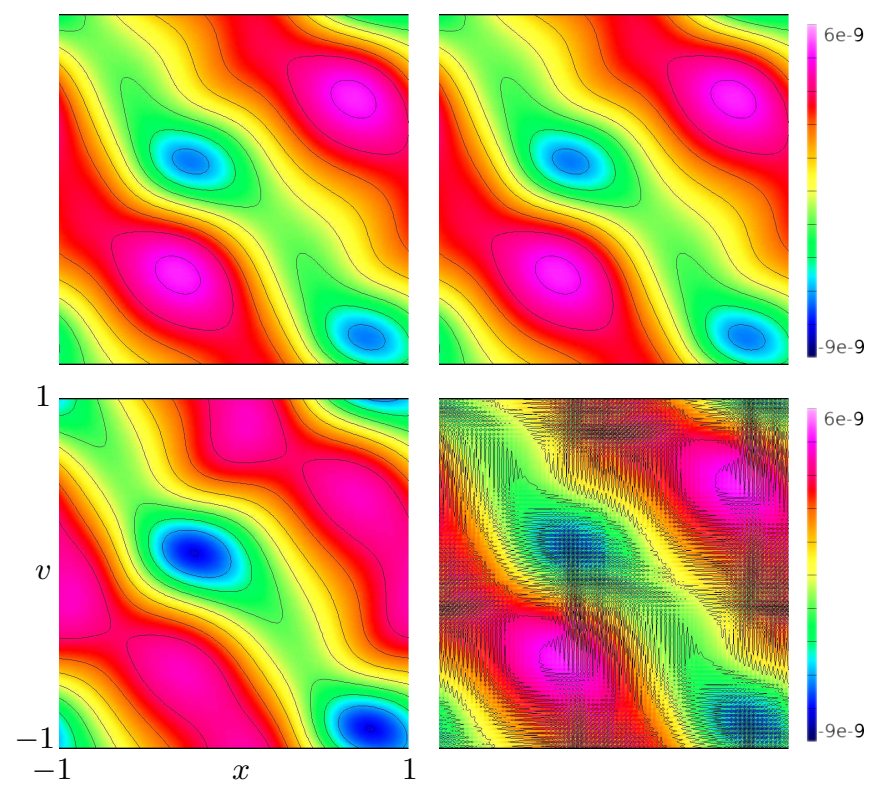

Fig. 4. Error in the manufactured solution using $N=160$ for the centered scheme (top left), new scheme (top right), artificial viscosity (bottom left), and PPM (bottom right). Note the larger errors from the artificial viscosity scheme and the noisy error signature of the PPM method.

exercises all the terms in the new algorithm and has a known, smooth exact solution. We solve

$$
\frac{\partial f}{\partial t}+\frac{\partial\left(v_{1} f\right)}{\partial x_{1}}+\frac{\partial\left(v_{2} f\right)}{\partial x_{2}}=\frac{\partial U}{\partial t}+\frac{\partial\left(v_{1} U\right)}{\partial x_{1}}+\frac{\partial\left(v_{2} U\right)}{\partial x_{2}}
$$

where

$$
\begin{aligned}
& v_{1}=a_{1} \sin \left(2 \pi x_{1}\right) \cos \left(2 \pi x_{2}\right)+v_{1,0}, \\
& v_{2}=a_{2} \sin \left(2 \pi x_{1}\right) \sin \left(2 \pi x_{2}\right)+v_{2,0}, \\
& U=a_{3} \sin \left(2 \pi x_{1}\right) \sin \left(2 \pi x_{2}\right) \cos (2 \pi t)+f_{0},
\end{aligned}
$$

and $a_{1}=0.1, a_{2}=0.2, a_{3}=0.3, v_{1,0}=1.0, v_{2,0}=0.9$, and $f_{0}=0.8$. Note that the right hand side of (18) forces the solution in such a way that $f=U$ is the solution to equation (18). Figure 4 shows the error for the various schemes using $N=160$. Table I shows convergence results for a series of resolutions.

A number of salient points can be made using this example. First, all schemes achieve the optimal fourth order convergence by $N=80$. Second, we note that the new scheme becomes

\begin{tabular}{||c||c|c|c|c||}
\hline \hline$N$ & centered & AV & PPM & new \\
\hline \hline 5 & $3.33 \mathrm{e}-2$ & $3.45 \mathrm{e}-2$ & $3.97 \mathrm{e}-2$ & $3.89 \mathrm{e}-2$ \\
\hline 10 & $3.44 \mathrm{e}-3$ & $4.12 \mathrm{e}-3$ & $3.36 \mathrm{e}-3$ & $3.44 \mathrm{e}-3$ \\
\hline 20 & $2.39 \mathrm{e}-4$ & $2.80 \mathrm{e}-4$ & $2.91 \mathrm{e}-4$ & $2.39 \mathrm{e}-4$ \\
\hline 40 & $1.63 \mathrm{e}-5$ & $1.93 \mathrm{e}-5$ & $2.18 \mathrm{e}-5$ & $1.63 \mathrm{e}-5$ \\
\hline 80 & $1.03 \mathrm{e}-6$ & $1.21 \mathrm{e}-6$ & $1.43 \mathrm{e}-6$ & $1.03 \mathrm{e}-6$ \\
\hline 160 & $6.41 \mathrm{e}-8$ & $7.53 \mathrm{e}-8$ & $9.14 \mathrm{e}-8$ & $6.41 \mathrm{e}-8$ \\
\hline 320 & $4.00 \mathrm{e}-9$ & $4.70 \mathrm{e}-9$ & $5.65 \mathrm{e}-9$ & $4.00 \mathrm{e}-9$ \\
\hline \hline
\end{tabular}

\section{TABLE I}

CONVERGENCE OF MAXIMUM POINTWISE ERROR FOR MANUFACTURED SOLUTION WITH VARIOUS SCHEMES. A RATIO OF 16 BETWEEN SUCCESSIVE ERRORS INDICATES FOURTH-ORDER CONVERGENCE.

virtually indistinguishable from the centered scheme at moderate resolutions, but provides sufficient non-linear viscosity when needed. Third, the mixing between low and high order in the new scheme is based on smooth, high-order-accurate smoothness indicators, and so the error is smooth. The error is not smooth for the PPM scheme, which uses hard switches to preserve accuracy near extrema; hard switches, also a feature of ENO schemes, tend to produce "noisy" errors.

\section{Additional Numerical Results}

In order to more clearly understand the character of the proposed scheme, we apply it to a number of well known test problems from the literature.

\section{A. Landau Damping}

We begin with the Landau damping problems [30, §8.6] with the initial distribution function given by

$$
f=\frac{1}{\sqrt{2 \pi}} \exp \left(-\frac{v^{2}}{2}\right)\left(1+\alpha \cos \left(\frac{x}{2}\right)\right)
$$

as specified in [4], [12]. We take the domain with $L=2 \pi$, $v_{\max }=2 \pi$. The parameter $\alpha$ defines the problem, with $\alpha=0.01$ often called "linear" and $\alpha=0.5$ "non-linear". For the weaker case with $\alpha=0.01$, the non-linear effects in the problem are negligible at early times, and so the use of a nonlinear scheme is unnecessary. As such, the desired behavior is that the oscillation-controlling methods produce results similar to those of the original centered scheme. Figure 5 shows the magnitude of the first and tenth Fourier modes of the electric field as well as a reference line indicating the analytic decay rate for the magnitude of the first Fourier mode. For the linear, PPM, and new schemes, the resonant frequency is computed to be $\omega=1.4155$ over the first twelve periods; the theoretical value is 1.4157 . The resonant frequency from the artificial viscosity scheme varies between $\omega=1.4155$ and $\omega=1.4661$ over the first twelve periods.

The artificial viscosity scheme also sits apart from the others in that it continually damps the solution, which eliminates the well-known recurrence phenomenon; note that the decay rate gradually departs from the analytical result as it becomes dominated by artificial damping. The centered scheme, the new scheme, and the PPM scheme behave in a similar way for the leading mode, however their treatment of the tenth 

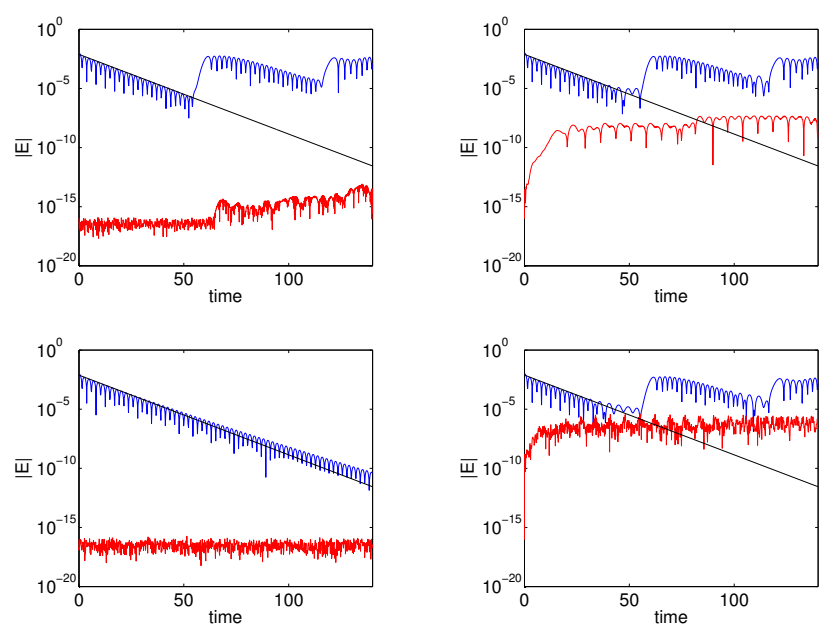

Fig. 5. Magnitude of the first (blue) and tenth (red) Fourier modes of the electric field for the Landau damping problem with $\alpha=0.01$. Also shown is a reference line indicating the theoretical decay rate of the first mode, $\gamma=-0.1553$. The results were computed on the mesh $N_{x}=N_{v}=64$ using the centered scheme (top left), the new scheme (top right), the centered scheme with artificial viscosity (bottom left), and PPM (bottom right).

mode is somewhat different. The non-linearity of the nonlinear schemes pushes energy into higher modes, even in the early-time, linear phase of the problem, and the PPM scheme transfers more energy than the new scheme. The original (linear) centered scheme shows no growth in the higher mode initially, but at longer times, the non-linearity of the VlasovPoisson system begins to transfer energy to higher modes. Unlike the (linear) artificial viscosity scheme, the centered scheme has too little dissipation to damp high-wavenumber modes, and the energy in higher modes will continue to grow, most likely causing instability.

For the Landau damping problem with $\alpha=0.5$, the nonlinearities in the problem will pose difficulties for the central scheme similar to those shown for the motivating two stream problem in Figure 1. In fact, this type of strongly non-linear example is the primary motivation of our investigation of non-linear limiting algorithms. The desired effect for this test problem is for the method to allow the representable non-linear features to grow but to provide sufficient damping to ensure overall algorithmic stability even at late time.

Figure 6 shows computed approximations at low resolution $\left(N_{x}=N_{v}=64\right)$ and late time $(t=140)$ and serves to demonstrate the need to include some form of artificial dissipation for this type of problem. It is clear that there is little of practical value that can be determined from the purely centered scheme (note that the full range of variation $[-0.14,0.65]$ is not visible with the unified color map), while the linear artificial viscosity scheme has essentially smeared any coherent structures in the problem. On the other hand, the two non-linear schemes, are able to capture the representable features in the problem; our new scheme does so with slightly better fidelity.

Figure 7 demonstrates this point by comparing the spatially averaged distribution functions of the coarse simulations with a more finely-resolved simulation computed using the new
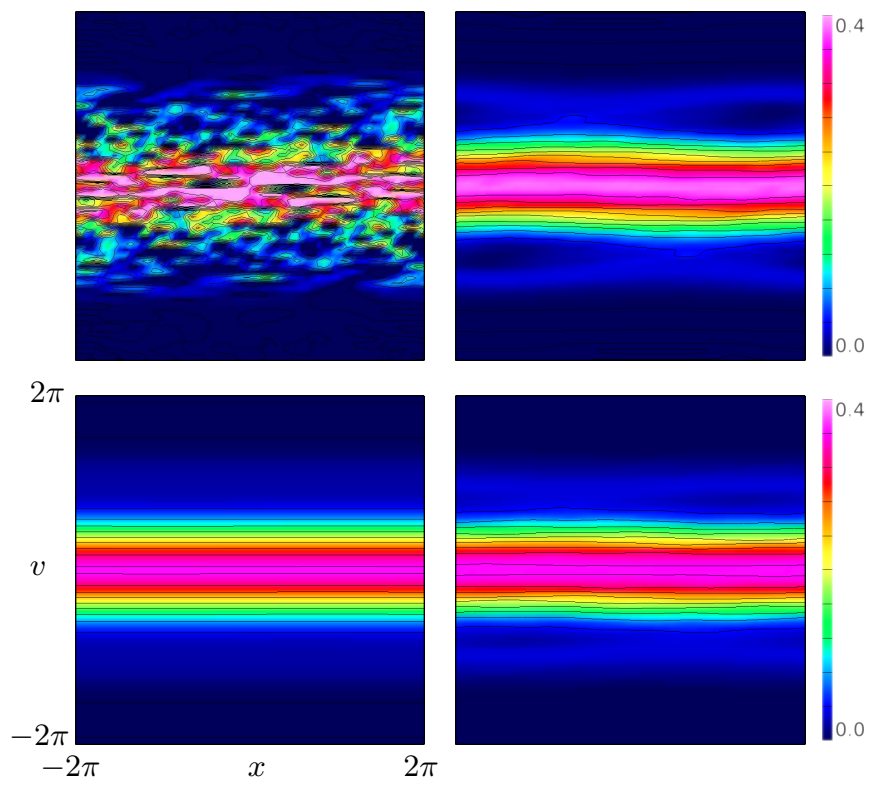

Fig. 6. Distribution function $f(x, v, t)$ at $t=140$ for the strong Landau damping problem with $\alpha=0.5$. The results were computed on the grid $N_{x}=N_{v}=64$ using the centered scheme (top left), new scheme (top right), the centered scheme with artificial viscosity (bottom left), and PPM (bottom right). Note that the range of variation for the unphysically oscillating solution using low-dissipation linear scheme is actually $[-0.14,0.65]$, so extrema have been clipped by the choice of color map. The new scheme does the best job capturing the trapping regions near $v= \pm 2$.
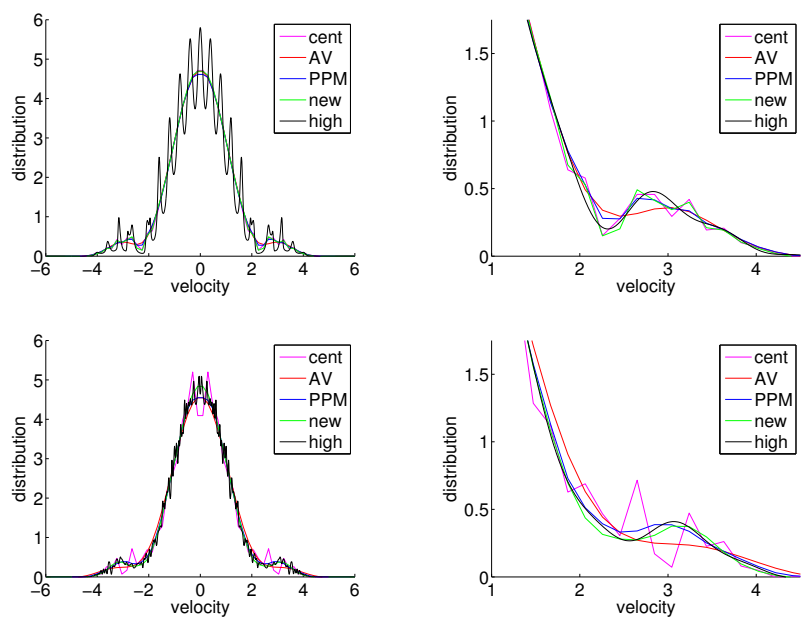

Fig. 7. Average distribution functions for the strong Landau damping problem at $t=30$ (top) and $t=140$ (bottom). The plots on the right include only the first 32 Fourier modes from the finely-resolved solution and are an enlargement near the shoulder in the distribution function to better show details. 


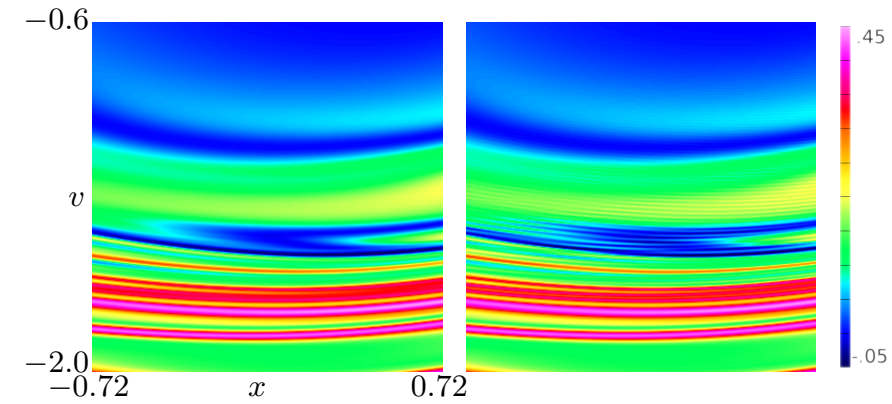

Fig. 8. Magnified region of the distribution function $f(x, v, t)$ at time $t=45$ for the two-stream instability problem using the mesh $N_{x}=N_{v}=2048$ and the new scheme (left) and the artificial viscosity scheme (right). Note the gridmode oscillations in the center of the plot on the right.

scheme with $N_{x}=N_{v}=1024$ at $t=30$ and $t=140$. Clearly, the fine scale structures in the problem will not be visible on the coarse mesh, and so for the zoomed-in plots on the right, we compare with only the fist 32 Fourier modes of the finely-resolved simulation. The artificial viscosity scheme clearly adds too much overall dissipation to the solution. At the early time $t=30$, the centered scheme and the new scheme are in good agreement, while the PPM scheme captures more of the variation but with greater amplitude error. At the later time, the centered scheme contains unacceptable unphysical oscillations. The PPM scheme and the new scheme, however, capture the general features of the smoothed, high-resolution solution with the new scheme generally showing slightly better agreement.

\section{B. Two-Stream Instability}

We return briefly to the motivating example of Section III. Throughout the results, we have mentioned that the artificial dissipation scheme is overly dissipative. One logical response would be to decrease the tunable dissipation coefficient $\mu$; the choice of such an algorithmic knob is always open to debate. However, Figure 8 shows the same computation as shown in Figure 3, but in a zoom near the origin, and compares the new scheme to the artificial viscosity scheme. Throughout this paper we have taken a constant value for the artificial viscosity parameter, and, in Figure 8, one can see that this choice is actually insufficient to suppress all numerical oscillations in the approximation at very high resolution. In the figure, note the unphysical, high-wavenumber oscillations in the artificial viscosity results near the top and bottom of the trapping region that are not present in the new scheme. Although small in magnitude, these oscillations show that our choice of the artificial viscosity parameter is not too high but is, rather, too small. In general, the choice of parameter is error prone and represents a severe disadvantage to linear artificial dissipation.

Finally, for quantitative comparison, we consider another variation of the two-stream instability problem with the initial distribution function given by

$$
f=f_{t}(v)(1+0.0005 \cos (0.2 x)),
$$

with

$f_{t}(v)=\frac{1}{\sqrt{8 \pi} v_{t}}\left[\exp \left(-\frac{\left(v-v_{0}\right)^{2}}{2 v_{t}^{2}}\right)+\exp \left(-\frac{\left(v+v_{0}\right)^{2}}{2 v_{t}^{2}}\right)\right]$,
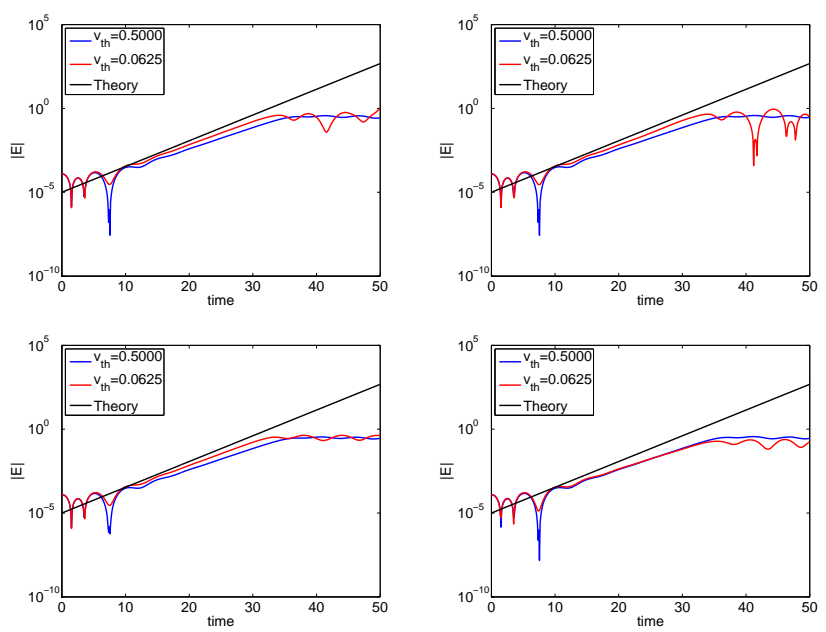

Fig. 9. Magnitude of the first Fourier mode of the electric field for the twostream instability problem with $v_{t}=0.5$ (blue) and $v_{t}=0.0625$ (red). Also shown is a reference line indicating the theoretical growth rate of the first mode, $\gamma=1 / \sqrt{8}$. The results were computed on the mesh $N_{x}=N_{v}=64$ using the centered scheme (top left), the new scheme (top right), the centered scheme with artificial viscosity (bottom left), and PPM (bottom right).

where $v_{0}=5 \sqrt{3} / 4$. The domain is defined by $L=5 \pi$ and $v_{\max }=8$, and we use $N_{x}=N_{v}=64$. Linear theory [30, $\left.\S 9.3\right]$ for cold distributions predicts a maximum growth rate of $\gamma=$ $1 / \sqrt{8} \approx 0.354$ will occur for mode $k=0.2$. Since we cannot represent delta functions discretely, we instead choose $v_{t}=$ $1 / 2$ and $1 / 16$ to observe the behavior as the initial distributions become narrower.

The results are plotted in Figure 9. We see that, for all schemes, the agreement is reasonable given the finite-width distributions and the asymptotic nature of the theoretical prediction. For all schemes except PPM, as we decrease the width of the initial streams, the growth rate increases towards the theoretical maximum value. We believe that the lack of change in the PPM scheme is due to its more severe reduction in order for poorly-resolved features leading to enhanced numerical damping. The new scheme does not have this problem, and in fact comes closest to the theoretical value: linearly extrapolating to the zero-width limit from the growth rates in the time range $20 \leq t \leq 30$, the zero-width growth rates are $0.178,0.180$, and 0.209 for the linear, artificial viscosity, and new schemes, respectively.

\section{Bump-on-Tail Instability}

As a final example, we address the bump-on-tail instability $[30, \S 9.4]$ using the parameters specified in [4], [12]. The initial distribution function is given by

$$
f=f_{b}(v)(1+0.04 \cos (0.3 x)),
$$

with

$$
f_{b}(v)=\frac{0.9}{\sqrt{2 \pi}} \exp \left(-\frac{v^{2}}{2}\right)+\frac{0.2}{\sqrt{2 \pi}} \exp \left(-4(v-4.5)^{2}\right) .
$$

The domain is defined by $L=10 \pi / 3$ and $v_{\max }=8$, and we use $N_{x}=128 m, N_{v}=512 \mathrm{~m}$, where $m$ is a parameter dictating the resolution. We have already demonstrated the 


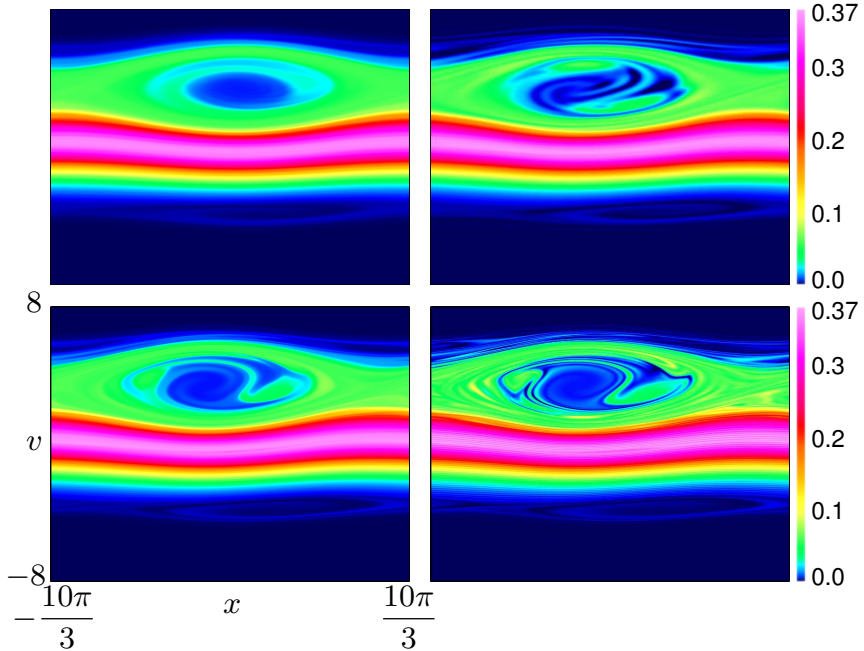

Fig. 10. Distribution function $f(x, v, t)$ at $t=200$ for the PPM scheme (left) and the new scheme (right) with grid resolutions $\left(N_{x}, N_{v}\right)=(128 m, 512 m)$ for $m=1$ (top) and $m=4$ (bottom). Note that the degree of detail in the PPM solution is comparable to the detail in the solution from the new scheme with four times less resolution. A quadratic ( $c f$. linear) color mapping was used in these plots to accentuate the details in the trapping region.
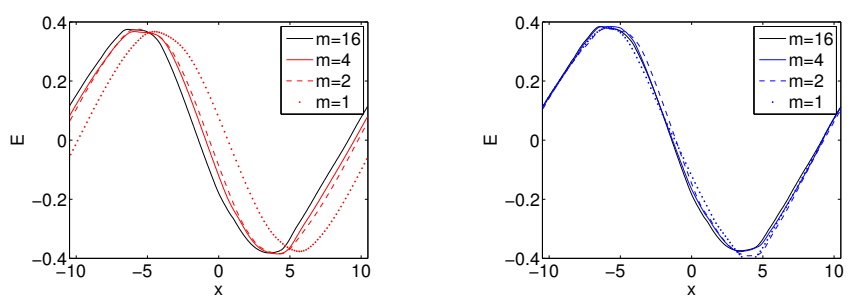

Fig. 11. Electric field $E(x, t)$ at $t=200$ for PPM scheme (left) and new scheme (right). The result from a highly resolved computation with $m=16$ is also displayed for reference. Note that the new scheme better captures the phase at all resolutions.

need to include viscosity into the approximation, and we have demonstrated that the linear artificial viscosity is not satisfactory. As a result we present results for this bump-ontail problem only for the new proposed scheme and the PPM scheme for comparison. Note that the computations have been performed with the other schemes, and the results present no surprises. Figure 10 shows computed approximations of the phase space distribution function at $t=200$ for $m=1$ and $m=4$ using the two schemes. Both approximation techniques capture the trapping region near $v=3$, but the new approximation (right) achieves significantly higher resolution of small features. In fact, the coarser results $(m=1)$ for the new scheme (top right) capture roughly the same set of features as the PPM scheme with four times as much resolution ( $m=4$, bottom left).

Notice further that the position of the trapped region is quite well located even at $m=1$ for the new scheme, while for PPM it is moving slightly too fast. This phase error can also be investigated via the electric field which is shown in Figure 11 for four resolutions, $m=1,2,4,16$. The final resolution is included as a reference and is intended to be a close approximation to the exact electric field. Here we see that the PPM scheme has accumulated a significant phase error for low resolution and is converging to the reference solution quite slowly. On the other hand the new scheme produces quite close results even at low resolution, and the higher resolutions are seen to be nicely convergent.

\section{CONCLUSIONS}

In this paper we have discussed the application of high-order finite-volume methods to the simulation of Vlasov systems. The need for the explicit or implicit inclusion of some form of artificial dissipation was demonstrated through a number of model problems including Landau damping, the two-stream instability, and the bump-on-tail instability. The standard methods used for comparison included high-order, linear artificial viscosity and the non-linear piecewise parabolic method. We introduced a new non-linear method that is designed to add an upwind artificial viscosity when the solution is underresolved, but to transition smoothly to a high-order, centered approximation for well-resolved regions of the flow. This method is constructed specifically with Vlasov systems in mind and leverages the specific type of non-linearities present in that the system disallows genuine non-linear discontinuities (i.e., shocks). The result is a scheme that behaves like a fourthorder, centered scheme when the solution is well-resolved, but adds an appropriate artificial dissipation as features in the solution become too fine to be represented accurately. The properties of this new scheme were demonstrated in relation to the other schemes through a series of classical test problems.

Two remaining important advantages of this new scheme deserve reiteration. First, the finite-volume method that lies at the heart of our algorithms are inherently local, and so parallelization is easily done. In fact, some computations presented in this paper used up to 512 processors, and nearly linear parallel scaling was observed. Second, the construction of the new method is quite general and extends to orders higher than fourth in a straightforward manner. That is to say, the recipe in Section VI is easily extensible to construct non-linear schemes of any even order that reduce to upwind schemes of one order less.

\section{ACKNOWLEDGEMENTS}

The authors would like to thank Dr. Bruce Cohen for his many helpful comments and suggestions.

This work was performed under the auspices of the U.S. Department of Energy by Lawrence Livermore National Laboratory under contract number DE-AC52-07NA27344. This work was funded by the Laboratory Directed Research and Development Program at LLNL under project tracking code 08-ERD-031. LLNL-JRNL-420843.

\section{REFERENCES}

[1] C. K. Birdsall and A. B. Langdon, Plasma Physics via Computer Simulation, 2nd ed., ser. The Adam Hilger Series on Plasma Physics. New York: Adam Hilger, 1991

[2] M. Shoucri and G. Knorr, "Numerical integration of the Vlasov equation,” J. Comput. Phys., vol. 14, no. 1, pp. 84-92, Jan. 1974.

[3] C. Z. Cheng and G. Knorr, "The integration of the Vlasov equation in configuration space," J. Comput. Phys., vol. 22, no. 3, pp. 330-351, Nov. 1976. 
[4] T. Nakamura and T. Yabe, "Cubic interpolated propagation scheme for solving the hyper-dimensional Vlasov-Poisson equation in phase space," Comput. Phys. Commun., vol. 120, pp. 122-154, 1999.

[5] A. J. Klimas, "A method for overcoming the velocity space filamentation problem in collisionless plasma model solutions," J. Comput. Phys. vol. 68, no. 1, pp. 202-226, Jan. 1987.

[6] T. Nakamura, R. Tanaka, T. Yabe, and K. Takizawa, "Exactly conservative semi-Lagrangian scheme for multi-dimensional hyperbolic equations with directional splitting technique," J. Comput. Phys., vol. 174, pp. 171-207, 2001.

[7] T. Yabe, Y. Ogata, K. Takizawa, T. Kawai, A. Segawa, and K. Sakurai, "The next generation CIP as a conservative semi-Lagrangian solver for solid, liquid, and gas," J. Comput. Appl. Math, vol. 149, pp. 267-277, 2002.

[8] F. Filbet, E. Sonnendrücker, and P. Bertrand, "Conservative numerical schemes for the Vlasov equation," J. Comput. Phys., vol. 172, pp. 166187,2001

[9] E. Fijalkow, "A numerical solution to the Vlasov equation," Comput. Phys. Commun., vol. 116, no. 2-3, pp. 319-328, Feb. 1999.

[10] N. Besse and E. Sonnendrücker, "Semi-Lagrangian schemes for the Vlasov equation on an unstructured mesh of phase space," J. Comput. Phys., vol. 191, pp. 341-376, 2003.

[11] M. Gutnic, M. Haefele, I. Paun, and E. Sonnendrücker, "Vlasov simulations on an adaptive phase space mesh," Comput. Phys. Commun., vol. 164, pp. 214-219, 2004.

[12] T. D. Arber and R. G. L. Vann, "A critical comparison of Euleriangrid-based Vlasov solvers," J. Comput. Phys., vol. 180, pp. 339-357, 2002.

[13] F. Filbet and E. Sonnendrücker, "Comparison of Eulerian Vlasov solvers," Comput. Phys. Commun., vol. 150, pp. 247-266, 2003.

[14] N. J. Sircombe and T. D. Arber, "VALIS: A split-conservative scheme for the relativistic 2D Vlasov-Maxwell system," J. Comput. Phys., vol. 228, no. 13, pp. 4773-4788, Jul. 2009.

[15] M. Kotschenreuther, G. Rewoldt, and W. M. Tang, "Comparison of initial value and eigenvalue codes for kinetic toroidal plasma instabilities," Comput. Phys. Commun., vol. 88, pp. 128-149, 1995.

[16] W. Dorland, F. Jenko, M. Kotschenreuther, and B. N. Rogers, "Electron temperature gradient turbulence," Phys. Rev. Lett., vol. 85, no. 26, pp. 5579-5582, Dec. 2000.

[17] J. Candy and R. E. Waltz, "An Eulerian gyrokinetic-Maxwell solver," $J$. Comput. Phys., vol. 182, no. 2, pp. 545-581, Apr. 2003.

[18] P. Colella and P. R. Woodward, "The Piecewise Parabolic Method (PPM) for gas-dynamical simulations," J. Comput. Phys., vol. 54, no. 1, pp. 174-201, Apr. 1984.

[19] P. Colella and M. D. Sekora, "A limiter for PPM that preserves accuracy at smooth extrema," J. Comput. Phys., vol. 227, pp. 7069-7076, 2008.

[20] C.-W. Shu, "Essentially non-oscillatory and weighted essentially nonoscillatory schemes for hyperbolic conservation laws," NASA Langley Research Center, Tech. Rep. NASA-CR-97-206253, Nov. 1997.

[21] P. Colella, M. R. Dorr, J. A. F. Hittinger, P. McCorquodale, and D. F. Martin, "High-order, finite-volume methods on locally-structured grids," in Numerical Modeling of Space Plasma Flows: Astronum 2008, ser Astronomical Society of the Pacific Conference Series, N. V. Pogorelov, E. Audit, P. Colella, and G. P. Zank, Eds., vol. 406. San Francisco: Astronomical Society of the Pacific, 2009, pp. 207-216.

[22] P. Colella, M. R. Dorr, J. A. F. Hittinger, and D. F. Martin, "High-order, finite-volume methods in mapped coordinates," J. Comput. Phys., 2010, submitted.

[23] S. T. Zalesak, "Fully multidimensional Flux-Corrected Transport algorithms for fluids," J. Comput. Phys., vol. 31, no. 2, pp. 335-362, Jun. 1979.

[24] B. Gustafsson, H.-O. Kreiss, and J. Oliger, Time Dependent Problems and Difference Methods, ser. Pure and Applied Mathematics. New York: John Wiley \& Sons, 1995

[25] J. P. Boyd, Chebyshev and Fourier Spectral Methods, 2nd ed. New York: Dover Publications, 2001.

[26] S. K. Godunov, "A difference scheme for numerical computation of discontinuous solutions of equations of fluid dynamics," Math. Sbornik, vol. 47, pp. 271-306, 1959, (In Russian).

[27] J. P. Boris and D. L. Book, "Flux-corrected transport. I. SHASTA, a fluid transport algorithm that works," J. Comput. Phys., vol. 11, no. 1, p. 38, Jan. 1973.

[28] B. Van Leer, "Towards the ultimate conservative difference scheme. V. A second-order sequel to Godunov's method," J. Comput. Phys., vol. 32 no. 1, pp. 101-136, Jul. 1979.
[29] A. K. Henrick, T. D. Aslam, and J. M. Powers, "Mapped weighted essentially non-oscillatory schemes: Achieving optimal order near critical points," J. Comput. Phys., vol. 207, pp. 542-567, 2005.

[30] N. A. Krall and A. W. Trivelpiece, Principles of Plasma Physics. New York: McGraw-Hill, 1973.

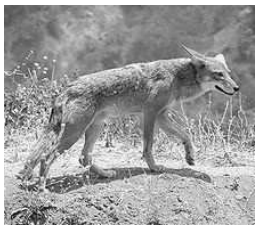

Jeffrey W. Banks Jeffrey Banks received the B.S., M.S., and Ph.D. degrees in Mathematics from Rensselaer Polytechnic Institute in Troy, NY, in 2002, 2002, and 2006, respectively.

He was a postdoctoral researcher in the Computational Sciences Research Institute at Sandia National Laboratory, Albuquerque, NM, from 2006 to 2008 and a postdoctoral researcher in the Center for Applied Scientific Computing at Lawrence Livermore National Laboratory from 2008 until 2009. Dr. Banks is currently a member of the technical staff at Lawrence Livermore National Laboratory working on numerical methods for hyperbolic and wave-dominated phenomena.

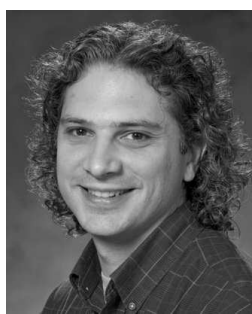

Jeffrey A. F. Hittinger In 1993, Jeffrey Hittinger received the B.S. degree in Mechanical Engineering from Lehigh University in Bethlehem, PA. He did his graduate studies at the University of Michigan, Ann Arbor, where he received the degrees of M.S.E. in Aerospace Engineering, M.S. in Applied Mathematics, and Ph.D. in Aerospace Engineering and Scientific Computing in 1994, 1997, and 2000, respectively.

From 2000 to 2002 , he was a postdoctoral researcher in the Center for Applied Scientific Computing at Lawrence Livermore National Laboratory. Dr. Hittinger is currently a member of the technical staff at Lawrence Livermore National Laboratory working on numerical methods for the simulation of laser-plasma interactions and of magnetically confined plasmas. 